\title{
The quantum Fourier transformation based on quantum vision representation
}

\author{
Hai-Sheng Li, Ping Fan, Hai-ying Xia, Shuxiang Song, Xiangjian He
}

\begin{abstract}
Quantum Fourier transform (QFT) plays a key role in many quantum algorithms, but the existing circuits of QFT are incomplete and lacking the proof of correctness. Furthermore, it is difficult to apply QFT to the concrete field of information processing. Thus, we firstly investigate quantum vision representation (QVR) and develop a model of QVR (MQVR). Then, we design four complete circuits of QFT and inverse QFT (IQFT) and describe the functions of their components. Meanwhile, we prove the correctness of the four complete circuits using formula derivation. Next, 2D QFT and 3D QFT based on QVR are proposed for the first time. Experimental results with simulation show the proposed QFTs are valid and useful in processing quantum images and videos. In conclusion, this paper develops a complete framework of QFT based on QVR and provides a feasible scheme for QFT to be applied in quantum vision information processing.
\end{abstract}

Index Terms-Quantum Fourier transformation, quantum information processing, quantum image processing, quantum computing.

\section{INTRODUCTION}

$\mathbf{I}$ $\mathrm{N}$ recent years, with the rapid development in many fields such as optical imaging, internet technology, high performance computing etc., the sizes of visual data have been increasing explosively. How to store and process the massive visual data efficiently is the key technology to be developed urgently. Quantum information processing (QIP) [1] uses qubits as its basic information units with two outstanding merits. One is that quantum storage capacity increases exponentially in comparison with the traditional storage as $n$ qubits can store $2^{n}$ data at the same time [2], and the other relies on the unique computing performances of quantum coherence, entanglement and superposition of quantum states. In fact, utilizing the unique properties, the algorithms, including Shor's discrete logarithms and integer factoring algorithms in the polynomial time [3], Deutsh's parallel computing algorithm with quantum parallelism and coherence [4], and Grover's quadratic speedup for unordered database search algorithm [5], are insurmountable so far by any known classical algorithms. We define a quantum vision information processing (QVIP) as a subfield of QIP in visual information, thus QVIP also has the above two merits and is a possible solution to store and process massive visual data efficiently.

Hai-Sheng Li (Corresponding author) is with College of Electronic Engineering, Guangxi Normal University, 541004, Guilin, Guangxi, P. R. China

Ping Fan is with College of Information Engineering, East China JiaoTong University, 330013, Nanchang, Jiangxi, P. R. China.

Hai-ying Xia and Shuxiang Song are with College of Electronic Engineering, Guangxi Normal University, 541004, Guilin, Guangxi, P. R. China.

Xiangjian $\mathrm{He}$ is with School of Electrical and Data Engineering, University of Technology Sydney, Australia.
QVIP mainly includes quantum image processing and quantum video processing. QVIP originated from the US Air Force Research Laboratory shows Grover's algorithm is applicable to image processing task, and describes Cybernet's modelbased vision system [6]. At present, the development of QVIP is mainly in two directions: research on quantum image representation and quantum transformation, and extension in the field of information processing.

The model of quantum image representation is a stored pattern by which data are stored in a quantum computer. The first of quantum image representation is called Qubit Lattice [7], [8], which stores a $2^{n} \times 2^{n}$ color image in quantum systems with $2^{2 n}$ qubits. The characteristic of Qubit Lattice is to represent a color using the frequency of an electromagnetic wave and convert the frequency into the amplitude of a onequbit quantum state by a physical device. Next, Le et al. [9] proposed a flexible representation of quantum images (FRQI), which stored a $2^{n} \times 2^{n}$ gray-scale image with $2 n+1$ qubits. To further improve the storage performance of quantum states, a normal arbitrary superposition state (NASS) was proposed [10], [11], which stored a $2^{n} \times 2^{n}$ color image with $2 n$ qubits. FRQI and NASS store the color information using amplitudes of quantum states, so they are suitable for an efficient quantum Fourier transform (QFT). For the convenience of color processing, a novel enhanced quantum representation (NEQR) was proposed to store a gray-scale value in 8-qubit states [12]. It is similar to classical color processing algorithms. Therefore, many classical image processing algorithms are more easily converted to their corresponding quantum algorithms. However, the key quantum algorithms, such as QFT and the quantum wavelet transform [13], are difficult to be directly applied to the image of NEQR. By adding a time dimension, quantum image representation can be expanded into quantum video representation, as in the FRQI version of quantum video representation [14], [15]. Through the above analysis, quantum image representation can be divided into two categories. The first category is to store color information using the amplitudes of a quantum state [16] - [20], and the second one is to store color information using the quantum states of multiple qubits [21] - [24].

QFT is one of the core algorithms of quantum information processing. For instance, QFT plays the key role in Shor's prime factorization and discrete logarithms [25]. Literatures [2], [26] - [29] have designed the quantum circuits of QFT, but these circuits are not complete, and lack the bit reversal circuit. In addition, the semiclassical Fourier transform [30] and the approximate Fourier transform [31] have been proposed. The complexity of the QFT implementation on $2^{n}$ elements is 
$O\left(n^{2}\right)$ [2]. In contrast, Fast Fourier Transform [32], one of the best classical algorithms, computes the discrete Fourier transform (DFT) with the complexity $O\left(n 2^{n}\right)$. QFT can exponentially speed up the computation of Fourier transforms than the Fourier transforms on a classical computer, but QFT is difficult to be applied in the concrete information processing field [2]. The emergence of the first category of quantum image representation makes it possible for QFT to be applied in image processing for compression, de-noising and encryption. The classical 2D and 3D DFTs can be applied directly to image processing [33], [34], but there are no counterparts for QVIP.

In the aspect of information processing, quantum image algorithms based on QFT are research hotspots, and their examples include the watermarking algorithms of quantum images [35], [36], the compression algorithm [10], and the encryption and decryption algorithm [37]. However, all of these algorithms are under the assumption that QFT is suitable for quantum information processing and has been already implemented.

The focus of this paper is to implement QFT based on QVR and provide a feasible scheme for QFT to be applied in the field of QVIP.

The contributions of this paper include:

- We firstly study a quantum vision representation (QVR) and build a model of QVR (MQVR), which is the foundation of applications of QFT on QVIP. As a typical example of quantum image representation of the first category, NASS [10] is extended into QVR.

- Then, we analyze the iteration formula of the classical Fourier transform by a generalized tensor product and design four complete circuits of QFT and inverse QFT (IQFT).

- Last, we design and implement 2D and 3D QFTs based on QVR, for the first time, that can be applied on quantum images and videos, respectively.

The rest of this paper is organized as follows. Section 2 introduces some related works. Section 3 describes the model of QVR of the first category. Section 4 designs the implementation circuits of QFT. Section 5 implements the 2D and 3D QFTs based on QVR. Experiment results with simulation are given in Section 6 and the conclusions are drawn in Section 7.

\section{THE GENERALIZED TENSOR PRODUCT}

In quantum computing, the tensor product, also called Kronecker product and denoted by $\otimes$, is a way of putting vector spaces together to form larger vector spaces. The tensor product is defined as follows. Let $A$ be an $n \times n$ matrix and $B$ be an $m \times m$ matrix, then the tensor product

$$
A \otimes B=\left[\begin{array}{ccc}
A_{0,0} B & \cdots & A_{0, n-1} B \\
\vdots & \ddots & \vdots \\
A_{n-1,0} B & \cdots & A_{n-1, n-1} B
\end{array}\right]
$$

is an $m n \times m n$ block matrix.

Suppose that $\mathscr{A}=\left\{A^{0}, A^{1}, \ldots, A^{m-1}\right\}$ and $\mathscr{B}=$ $\left\{B^{0}, B^{1}, \ldots, B^{n-1}\right\}$ are two sets of matrices, where $A^{i}$ is an $n \times n$ matrix, $0 \leq i<m$, and $B^{j}$ is an $m \times m$ matrix, $0 \leq j<n$. Then, the generalized tensor product $C=\mathscr{A} \otimes \mathscr{B}$ is an $m n \times m n$ matrix and can be calculated by $C_{u m+w, v m+z}=a_{u, v}^{w} b_{w, z}^{v}$ with $0 \leq u, v<n, 0 \leq w, z<m$, where $C_{u m+w, v m+z}, a_{u, v}^{w}$ and $b_{w, z}^{v}$ are the elements of matrices $C, A^{w}$ and $B^{v}$, respectively. More details are described in references [38], [39].

Next, we introduce the perfect shuffle permutation to factorize the generalized tensor product. Let $P_{n, m}$ be an $m n \times m n$ matrix of the perfect shuffle permutation, then $P_{n, m}$ satisfies that $\left(P_{n, m}\right)_{k, l}=\delta_{v, z^{\prime}} \delta_{z, v^{\prime}}$ where $k=v n+z, l=v^{\prime} m+z^{\prime}$, $0 \leq v, z^{\prime}<m, 0 \leq v^{\prime}, z<n, \delta_{x, y}$ is the Kronecker delta function, i.e., $\delta_{x, y}=0$ if $x \neq y$, otherwise $\delta_{x, y}=1$.

$P_{n, m}$ shuffles $n$ packs of $m$ cards into $m$ packs of $n$ cards. If $A^{i}$ and $B^{j}$ are unitary matrices, then $\mathscr{A} \otimes \mathscr{B}$ is a unitary matrix and can be factorized into

$$
\mathscr{A} \otimes \mathscr{B}=P_{m, n} \operatorname{Diag}(\mathscr{A}) P_{n, m} \operatorname{Diag}(\mathscr{B}),
$$

where $\operatorname{Diag}(\mathscr{A})=\operatorname{Diag}\left(A^{0}, A^{1}, \ldots, A^{m-1}\right) \quad$ and $\operatorname{Diag}(\mathscr{B})=\operatorname{Diag}\left(B^{0}, B^{1}, \ldots, B^{n-1}\right)$ are block diagonal matrices [39].

In order to build the relatively complete system of a generalized tensor product, some new concepts are defined as follows.

Definition 1. Let $\mathscr{A}=\left\{A^{0}, A^{1}, \ldots, A^{m-1}\right\}$ and $\mathscr{D}=\left\{D^{0}, D^{1}, \ldots, D^{m-1}\right\}$ be two sets of matrices where $A^{i}$ and $D^{i}$ are $n \times n$ matrices. Then, the generalized product is defined as $\mathscr{A} \times \mathscr{D}=\mathscr{A} \mathscr{D}=$ $\left\{A^{0} \times D^{0}, A^{1} \times D^{1}, \ldots, A^{m-1} \times D^{m-1}\right\}$.

Definition 2. The transpose, conjugate transpose and inverse of the matrix set $\mathscr{A}$ are defined as follows:

$$
\left\{\begin{array}{l}
\mathscr{A}^{T}=\left\{\left(A^{0}\right)^{T},\left(A^{1}\right)^{T}, \ldots,\left(A^{m-1}\right)^{T}\right\}, \\
\mathscr{A}^{+}=\left\{\left(A^{0}\right)^{+},\left(A^{1}\right)^{+}, \ldots,\left(A^{m-1}\right)^{+}\right\}, \\
\mathscr{A}^{-1}=\left\{\left(A^{0}\right)^{-1},\left(A^{1}\right)^{-1}, \ldots,\left(A^{m-1}\right)^{-1}\right\},
\end{array}\right.
$$

where $\left(A^{i}\right)^{T},\left(A^{i}\right)^{+}$and $\left(A^{i}\right)^{-1}$ denote the transpose, conjugate transpose and inverse of the matrix $A^{i}$, respectively.

The following equations hold using equations (2), definitions 1 and 2.

$$
\begin{aligned}
(\mathscr{A} \otimes \mathscr{B})^{T} & =P_{m, n}\left(\mathscr{B}^{T} \otimes \mathscr{A}^{T}\right) P_{n, m}, \\
(\mathscr{A} \otimes \mathscr{B})^{+} & =P_{m, n}\left(\mathscr{B}^{+} \otimes \mathscr{A}^{+}\right) P_{n, m}, \\
(\mathscr{A} \otimes \mathscr{B})^{-1} & =P_{m, n}\left(\mathscr{B}^{-1} \otimes \mathscr{A}^{-1}\right) P_{n, m} .
\end{aligned}
$$

Let $\mathscr{A}$ and $\mathscr{C}$ be two sets of matrices containing $m$ matrices with size $n \times n, \mathscr{B}$ and $\mathscr{D}$ be two sets of matrices containing $n$ matrices with size $m \times m, I_{m}$ and $I_{n}$ be $m \times m$ and $n \times n$ identity matrices, respectively. Then, the following equation holds [38]:

$$
(\mathscr{A} \times \mathscr{C}) \otimes(\mathscr{B} \times \mathscr{D})=\left(\mathscr{A} \otimes I_{m}\right) \times(\mathscr{C} \otimes \mathscr{B}) \times\left(I_{n} \otimes \mathscr{D}\right)
$$




\section{MQVR}

MQVR is the stored pattern by which visual data are stored in a quantum computer, and is the foundation of applications of QFT on QVIP. We introduce MQVR by extending NASS into a quantum vision representation. Firstly, we briefly describe NASS.

\section{A. The representation of a quantum image NASS}

An angle set can be used to represent a gray-scale set or a color set in the RGB model:

$$
\phi_{i}=\frac{i \pi}{2(M-1)}
$$

and

$$
\mathscr{Y}=\left\{\phi_{0}, \ldots, \phi_{M-1}\right\},
$$

where $0 \leq i<M$. For a gray-scale set, $M=256$, and $\phi_{i}$ corresponds to the gray-scale value $i$. For an RGB color set, $M=2^{24}$, and $\phi_{i}$ corresponds to a RGB color $(r, g, b)$ and $i=256 \times 256 \times r+256 \times g+b$.

A NASS state $|\psi\rangle$ can store colors and coordinates of $2^{n}$ pixels:

$$
|\psi\rangle=\sum_{i=0}^{2^{n}-1} \theta_{i}|i\rangle=\sum_{i=0}^{2^{n}-1} \theta_{i}\left|i_{n} \cdots i_{2} i_{1}\right\rangle,
$$

where $\theta_{i}=a_{i} / \sqrt{\sum_{j=0}^{2^{n}-1} a_{j}^{2}}$ and $|i\rangle=\left|i_{n} \cdots i_{2} i_{1}\right\rangle$ are the color and coordinate of the $i$-th pixel. Here, $a_{i} \in \mathscr{Y}$ in equation (8), and $i=\sum_{j=1}^{n} i_{j} \times 2^{j-1}$ and $i_{j} \in\{0,1\}$, i.e., $i_{n} \cdots i_{2} i_{1}$ is the binary expansion of integer $i$.

Substituting $|i\rangle=\left|x_{m}\right\rangle\left|y_{k}\right\rangle$ into equation (9), we obtain the NASS state $\left|\psi_{2}\right\rangle$ of an image

$$
\begin{aligned}
\left|\psi_{2}\right\rangle & =\sum_{i=0}^{2^{n}-1} \theta_{i}\left|x_{m}\right\rangle\left|y_{k}\right\rangle \\
& =\sum_{i=0}^{2^{n}-1} \theta_{x_{m}, y_{k}}\left|i_{n} \ldots i_{k+1}\right\rangle\left|i_{k} \ldots i_{1}\right\rangle,
\end{aligned}
$$

where $\left|x_{m}\right\rangle=\left|i_{n} \ldots i_{k+1}\right\rangle$ and $\left|y_{k}\right\rangle=\left|i_{k} \ldots i_{1}\right\rangle$ are the $\mathrm{X}$ axis and Y-axis of the image, and $n=m+k$. More details are shown in our previous work [10].

\section{B. The representation of a quantum video}

Substituting $|i\rangle=\left|x_{m}\right\rangle\left|y_{k}\right\rangle\left|t_{h}\right\rangle$ into equation (9), we obtain the NASS state $\left|\psi_{3}\right\rangle$ of a video

$$
\begin{aligned}
\left|\psi_{3}\right\rangle & =\sum_{i=0}^{2^{n}-1} \theta_{i}\left|x_{m}\right\rangle\left|y_{k}\right\rangle\left|t_{h}\right\rangle \\
& =\sum_{i=0}^{2^{n}-1} \theta_{x_{m}, y_{k}, t_{h}}\left|i_{n} \ldots i_{h+k+1}\right\rangle\left|i_{h+k} \ldots i_{h+1}\right\rangle\left|i_{h} \ldots i_{1}\right\rangle,
\end{aligned}
$$

where $\left|x_{m}\right\rangle,\left|y_{k}\right\rangle$ and $\left|t_{h}\right\rangle$ are the $\mathrm{X}$-axis, Y-axis and time-axis of a video, and $n=m+k+h$.

Thus, we have constructed MQVR based on NASS to store visual data including images and videos. For instance, the NASS state

$$
\left|\psi_{2}\right\rangle=\theta_{0,0}|000\rangle|00\rangle+\theta_{0,1}|000\rangle|01\rangle+\cdots+\theta_{7,3}|111\rangle|11\rangle
$$

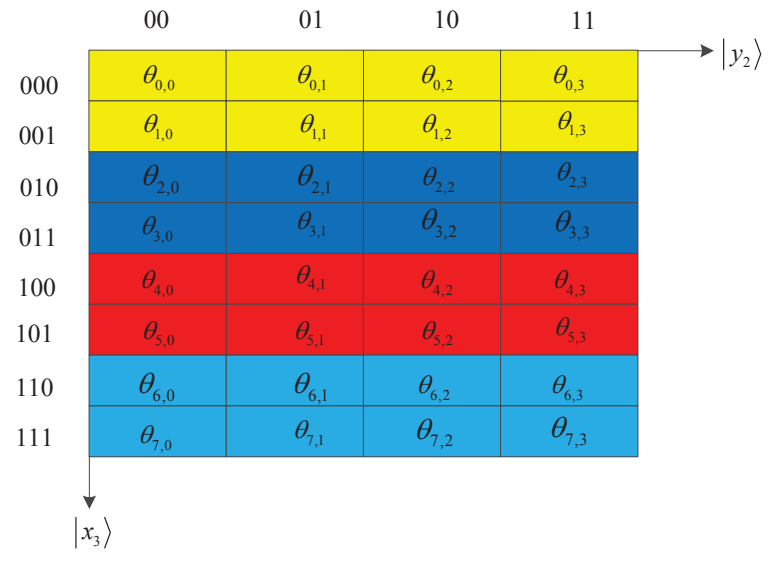

Fig. 1. A sample color image.

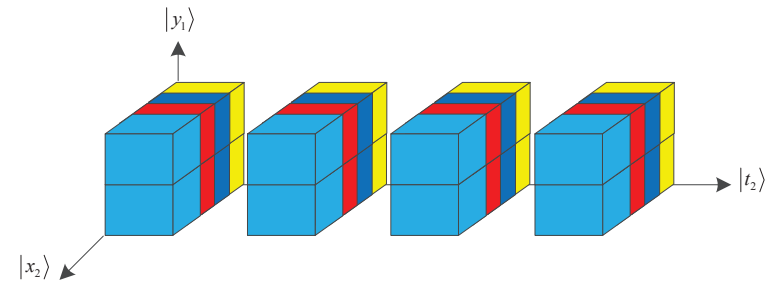

Fig. 2. A sample video with 4 frames.

represents the color image of $8 \times 4$ (height multiplies weight) shown in Fig. 1.

The NASS state

$$
\begin{array}{r}
\left|\psi_{3}\right\rangle=\theta_{0,0,0}|00\rangle|0\rangle|00\rangle+\theta_{0,0,1}|00\rangle|0\rangle|01\rangle \\
+\cdots+\theta_{3,1,3}|11\rangle|1\rangle|11\rangle
\end{array}
$$

represents the video with four frames shown in Fig. 2 where each frame is a $4 \times 2$ image.

The same string can have different meanings for different data types in classic computers. For instance, a binary string 0100001 can express a char 'A' or a number 65 . Similarly, using the circuit in reference [10], we can store an image (shown in Fig. 1 ) or a video (shown in Fig. 2 ) in the following state

$$
|\psi\rangle=\sum_{i=0}^{2^{5}-1} \theta_{i}|i\rangle .
$$

Meanwhile, the priori knowledge ' $x_{3}, y_{2}$ ' or ' $x_{2}, y_{1}, t_{2}$ ' is equivalently a data type, implying an image or a video stored in the state $|\psi\rangle$.

\section{THE QUANTUM FOURIER TRANSFORM}

Quantum computing can be implemented by using quantum gates, and universal quantum gates can be expressed as combinations of single-qubit and two-qubit gates. We introduce some of base gates and their corresponding matrices shown in Fig.3. The identity $I_{2}$, Hadamard $H$ and Swap gates are well-known and can be found in reference [2]. The gate $U$ corresponds to a unitary matrix. $I_{2}$ and $H$ are two examples of a unitary matrix $U$. The $2^{n} \times 2^{n}$ identity matrix $\left(I_{2}\right)^{\otimes n}=I_{2^{n}}$ denotes the circuit of $n$ qubits. 


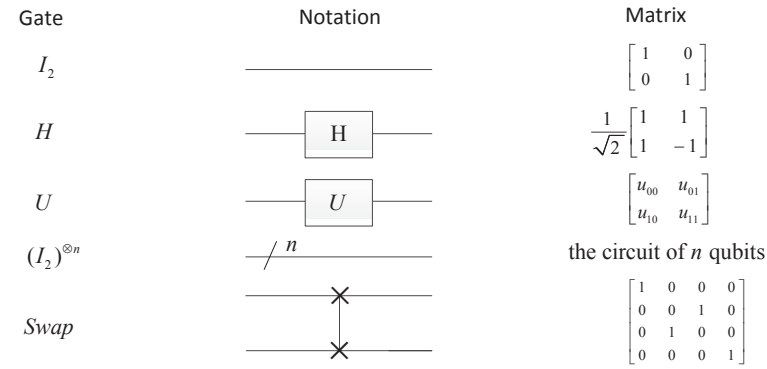

Fig. 3. Notations for some of base gates with their corresponding matrices.

The QFT's $F_{2^{n}}$ on $2^{n}$ elements is defined by [41]

$$
F_{2^{n}}:|k\rangle \rightarrow \frac{1}{\sqrt{2^{n}}} \sum_{j=0}^{2^{n}-1} e^{2 \pi i j k / 2^{n}}|j\rangle,
$$

where $i$ is the imaginary unit, and $k, j \in\left[0,2^{n}-1\right]$.

Acting on the quantum state $|\psi\rangle$ in equation (9), the result is

$$
F_{2^{n}}|\psi\rangle=\sum_{k=0}^{2^{n}-1} \alpha_{k}|k\rangle,
$$

where $\alpha_{k}=\frac{1}{\sqrt{2^{n}}} \sum_{j=0}^{2^{n}-1} \theta_{k} e^{2 \pi i j k / 2^{n}}$. Its corresponding matrix form is

$$
F_{2^{n}}\left[\begin{array}{lll}
\theta_{0} & \cdots & \theta_{2^{n}-1}
\end{array}\right]^{T}=\left[\begin{array}{lll}
\alpha_{0} & \cdots & \alpha_{2^{n}-1}
\end{array}\right]^{T} .
$$

By using the iteration formula of the Fourier transform in [36], the iteration formulas of QFT and IQFT can be given

$$
\begin{gathered}
F_{2^{n}}=\left(\left\{F^{0}, F^{1}, \ldots, F^{2^{n-1}-1}\right\} \otimes F_{2^{n-1}}\right) P_{2^{n-1}, 2} \\
\left(F_{2^{n}}\right)^{-1}=\left(\left\{\left(F^{0}\right)^{-1},\left(F^{1}\right)^{-1}, \ldots,\left(F^{2^{n-1}-1}\right)^{-1}\right\}\right. \\
\left.\otimes\left(F_{2^{n-1}}\right)^{-1}\right) P_{2^{n-1}, 2}
\end{gathered}
$$

where $P_{2^{n-1}, 2}$ is a perfect shuffle permutation, the initial value $F_{2}=H, n \geq 2$ and

$$
F^{k}=\frac{\sqrt{2}}{2}\left[\begin{array}{cc}
1 & e^{2 \pi i k / 2^{n}} \\
1 & -e^{2 \pi i k / 2^{n}}
\end{array}\right], k=0,1, \ldots, 2^{n-1}-1 .
$$

Two special matrices of the perfect shuffle permutation $P_{2^{n-1}, 2}$ and $P_{2,2^{n-1}}$ act on the quantum state $\left|j_{n} \ldots j_{2} j_{1}\right\rangle$ of $n$ qubits:

$$
\begin{aligned}
P_{2^{n-1}, 2}\left|j_{n} \ldots j_{2} j_{1}\right\rangle & =\left|j_{1} j_{n} \ldots j_{2}\right\rangle, \\
P_{2,2^{n-1}}\left|j_{n} j_{n-1} \ldots j_{1}\right\rangle & =\left|j_{n-1} \ldots j_{1} j_{n}\right\rangle .
\end{aligned}
$$

Thus, we derive their iterative equations

$$
P_{2^{n-1}, 2}=\left(P_{2^{n-2}, 2} \otimes I_{2}\right)\left(I_{2^{n-2}} \otimes S w a p\right)
$$

and

$$
P_{2,2^{n-1}}=\left(I_{2} \otimes P_{2,2^{n-2}}\right)\left(\operatorname{Swap} \otimes I_{2^{n-2}}\right)
$$

with the initial values of the iteration $P_{2,2}=S w a p$, where the swap gate Swap, identity gate $I_{2}$ and $I_{2^{n-2}}=\left(I_{2}\right)^{\otimes n-2}$ are shown in Fig.3. We design the implement circuits of $P_{2^{n-1}, 2}$ and $P_{2,2^{n-1}}$ with the complexity of $O(n)$ shown in Fig.4.

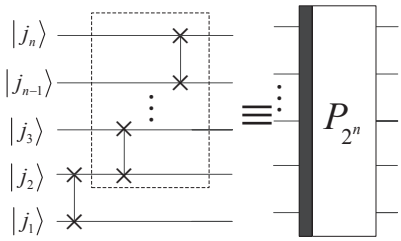

(a) $P_{2^{n-1}, 2}$ (b) $P_{2,2^{n-1}}$

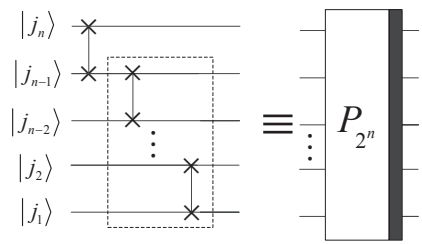

Fig. 4. The implement circuits of $P_{2^{n-1}, 2}$ and $P_{2,2^{n-1}}$. In (a), the right one corresponds to the abbreviation notation of $P_{2,2^{n-1}}$, and the dotted box is the circuit of $P_{2^{n-2}, 2}$; In (b), the right one is the abbreviation notation of $P_{2,2^{n-1}}$, and the dotted box is the circuit of $P_{2,2^{n-2}}$.

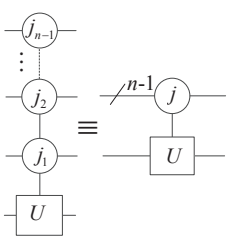

(a) $C_{n-1}^{j}(U)$

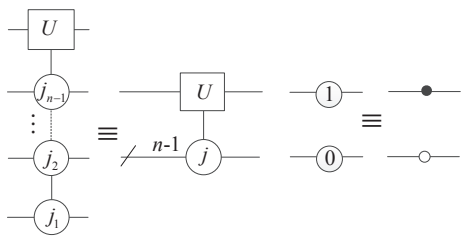

(b) $\quad V_{n-1}^{j}(U)$ (c) Control qubit
Fig. 5. Quantum controlled- $U$ gates of $n$-qubits. The equivalent representation of the control qubit is shown in (c).

Quantum gates are the same in Figs.4 (a) and (b), but these quantum gates are in reverse order. Therefore, the results are the same by running circuits (a) and (b) in the direction of the black box. We also adopt similar abbreviation notations to denote the circuits which are composed of the same quantum gates with reverse order in the following sections.

In order to facilitate the implementation of QFT in (18), two controlled- $U$ gates of $n$-qubits $C_{n-1}^{j}(U)$ and $V_{n-1}^{j}(U)$ are defined by

$$
C_{n-1}^{j}(U)=(|j\rangle\langle j|) \otimes U+\sum_{i=0, i \neq j}^{2^{n-1}-1}\left((|i\rangle\langle i|) \otimes I_{2}\right)
$$

and

$$
V_{n-1}^{j}(U)=(U \otimes|j\rangle\langle j|)+\sum_{i=0, i \neq j}^{2^{n-1}-1}\left(I_{2} \otimes(|i\rangle\langle i|)\right) .
$$

The notations of $C_{n-1}^{j}(U)$ and $V_{n-1}^{j}(U)$ are shown in Fig.5, where $j_{n-1} \cdots j_{2} j_{1}$ is the binary expansion of integer $j$, and numbers 1 and 0 represent the black and white points of the control qubit in the controlled gates (see (c) of Fig.5).

Suppose that the matrix set $\mathscr{A}=\left\{A^{0}, A^{1}, \ldots, A^{2^{n}-1}\right\}$ satisfies $A^{i}=I_{2}$ for $i \neq j$, then we have

$$
\mathscr{A} \otimes I_{2^{n-1}}=A^{j} \otimes(|j\rangle\langle j|)+\sum_{i=0, i \neq j}^{2^{n-1}-1} I_{2} \otimes(|i\rangle\langle i|)=V_{n-1}^{j}\left(A^{j}\right)
$$

and

$$
I_{2^{n-1}} \otimes \mathscr{A}=(|j\rangle\langle j|) \otimes A^{j}+\sum_{i=0, i \neq j}^{2^{n-1}-1}(|i\rangle\langle i|) \otimes I_{2}=C_{n-1}^{j}\left(A^{j}\right) .
$$

We can derive two useful equations from (6):

$\mathscr{A} \otimes \mathscr{D}=\left(\mathscr{A} \times I_{n}\right) \otimes\left(I_{m} \times \mathscr{D}\right)=\left(\mathscr{A} \otimes I_{m}\right)\left(I_{n} \otimes \mathscr{D}\right)$, 


$$
(\mathscr{A} \times \mathscr{C}) \otimes I_{m}=\left(\mathscr{A} \otimes I_{m}\right)\left(\mathscr{C} \otimes I_{m}\right) .
$$

Let $R_{n}=\left[\begin{array}{cc}1 & 0 \\ 0 & e^{2 \pi i / 2^{n}}\end{array}\right], F^{k}$ in (20) is rewritten as

$$
F^{k}=H \times\left(R_{n}\right)^{k} .
$$

By using equations (29), (30) and (31), equation (18) is rewritten as

$$
F_{2^{n}}=\left(H \otimes I_{2^{n-1}}\right) S_{2^{n}}\left(R_{n}\right)\left(I_{2} \otimes F_{2^{n-1}}\right) P_{2^{n-1}, 2}
$$

and

$$
S_{2^{n}}\left(R_{n}\right)=\left\{I_{2}, R_{n},\left(R_{n}\right)^{2}, \ldots,\left(R_{n}\right)^{2^{n-1}-1}\right\} \otimes I_{2^{n-1}}
$$

with initial values of the iteration $P_{2,2}=$ Swap, $F_{2}=H$ and $S_{4}\left(R_{2}\right)=\left\{I_{2}, R_{2}\right\} \otimes I_{2}$, where $\left(R_{n}\right)^{i}$ is the $i$-th power of the matrix $R_{n}$.

According to the analysis of the difference between equations (18) and (19), we obtain the iteration of IQFT by replacing $R_{n}$ with $\left(R_{n}\right)^{-1}$ in (32)

$$
\begin{aligned}
\left(F_{2^{n}}\right)^{-1}=\left(H \otimes I_{2^{n-1}}\right) S_{2^{n}}\left(\left(R_{n}\right)^{-1}\right) & \\
& \times\left(I_{2} \otimes\left(F_{2^{n-1}}\right)^{-1}\right) P_{2^{n-1}, 2} .
\end{aligned}
$$

Next, we describe the four circuits of QFT in detail.

\section{A. The first circuit of $Q F T$}

We have the following proposition to implement the first circuit of QFT.

Proposition 1. The iteration equation of $S_{2^{n}}\left(R_{n}\right)$ is

$$
\begin{aligned}
S_{2^{n}}\left(R_{n}\right)= & \left(R_{n} \otimes I_{2^{n-2}} \otimes|1\rangle\left\langle 1\left|+I_{2^{n-1}} \otimes\right| 0\right\rangle\langle 0|\right) \\
& \times S_{2^{n-1}}\left(R_{n-1}\right) \otimes I_{2} .
\end{aligned}
$$

Proof. By using equations (27), (30) and (33), we obtain

$$
\begin{aligned}
& S_{2^{n}}\left(R_{n}\right) \\
& =\left(\left\{I_{2}, R_{n}, I_{2}, R_{n}, \ldots, I_{2}, R_{n}\right\} \otimes I_{2^{n-1}}\right) \\
& \times\left(\left\{I_{2}, I_{2},\left(R_{n}\right)^{2},\left(R_{n}\right)^{2}, \ldots,\left(R_{n}\right)^{2^{n-1}-2}\right\} \otimes I_{2^{n-1}}\right) \\
& =\left(\prod_{j \text { isodd }} V_{n-1}^{j}\left(R_{n}\right)\right)\left(S_{2^{n-1}}\left(\left(R_{n}\right)^{2}\right) \otimes I_{2}\right) .
\end{aligned}
$$

$j$ is odd when $j=i_{n-1} \cdots i_{2} 1$, thus the circuit of $\prod_{\text {is }} V_{n-1}^{j}\left(R_{n}\right)$ is shown in Fig.6. The left circuit can be simplified to the right circuit in Fig.6 and it equals with a tensor product in (37).

$$
\begin{aligned}
& \prod_{j \text { isodd }} V_{n-1}^{j}\left(R_{n}\right) \\
& =R_{n} \otimes I_{2^{n-2}} \otimes|1\rangle\left\langle 1\left|+I_{2^{n-1}} \otimes\right| 0\right\rangle\langle 0|
\end{aligned}
$$

Substituting (37) and $\left(R_{n}\right)^{2}=R_{n-1}$ into (36), we obtain the equation (35).

We design the quantum circuit of QFT in equations (32) and (33) with the complexity of $O\left(n^{2}\right)$ shown in Fig.7 by using Proposition 1 and the circuit of $P_{2^{n-1}, 2}$ in Fig.4.

To facilitate designs of three other circuits of QFT, we define

$$
F_{2^{m}}^{2}=F_{2^{m}}^{3}=F_{2^{m}}^{4}=F_{2^{m}},
$$

where $F_{2^{m}}^{i}$ corresponds to the $i$-th circuit of QFT, $i \in\{2,3,4\}$ and $m \in\{2,3, \ldots, n\}$.

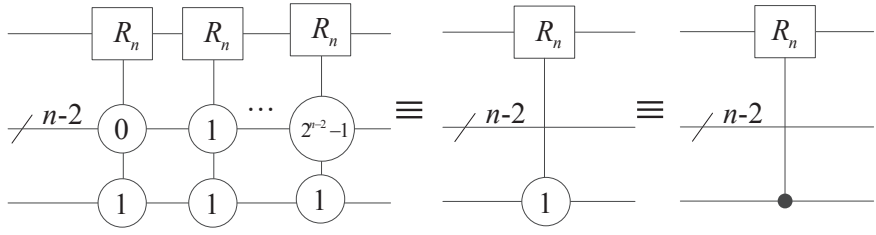

Fig. 6. The circuit of $\prod_{j \text { is odd }} V_{n-1}^{j}\left(R_{n}\right)$. The circuit of $V_{n-1}^{j}\left(R_{n}\right)$ is shown in Fig.5 (b) by replacing the matrix $U$ with $R_{n}$.

\section{B. The second circuit of $Q F T$}

The inversion of IQFT in (34) is given

$$
\begin{aligned}
F_{2^{n}}^{2} & =F_{2^{n}}=\left[\left(F_{2^{n}}\right)^{-1}\right]^{-1} \\
& =\left\{\left(H \otimes I_{2^{n-1}}\right)\left[I_{2} \otimes\left(F_{2^{n-1}}\right)^{-1}\right] P_{2^{n-1}, 2}\right\}^{-1} \\
& =P_{2,2^{n-1}}\left(I_{2} \otimes F_{2^{n-1}}\right) S_{2^{n}}\left(R_{n}\right)\left(H \otimes I_{2^{n-1}}\right) \\
& =P_{2,2^{n-1}}\left(I_{2} \otimes F_{2^{n-1}}^{2}\right) S_{2^{n}}\left(R_{n}\right)\left(H \otimes I_{2^{n-1}}\right)
\end{aligned}
$$

with same initial values of the iteration in equations (32) and (33), and its implementation circuit is shown in Fig.8. The quantum gates are the same with reverse order in Fig.7 and Fig.8, so the two abbreviation notations of QFT are similar with that of the perfect shuffle permutations in Fig.4.

\section{The third circuit of QFT}

We define

$$
T_{2^{n}}\left(R_{n}\right)=I_{2^{n-1}} \otimes\left\{\left(R_{n}\right)^{0},\left(R_{n}\right)^{1}, \ldots,\left(R_{n}\right)^{2^{n-1}-1}\right\}
$$

and have the following proposition to implement the third circuit of the QFT .

Proposition 2. The iteration equation of $T_{2^{n}}\left(R_{n}\right)$ is

$$
\begin{aligned}
T_{2^{n}}\left(R_{n}\right)= & {\left[I_{2^{n-2}} \otimes\left(|1\rangle\left\langle 1\left|\otimes R_{n}+\right| 0\right\rangle\langle 0| \otimes I_{2}\right)\right] } \\
& \times T_{2^{n-1}}\left(I_{2} \otimes R_{n-1}\right)
\end{aligned}
$$

with the initial value $T_{2^{2}}\left(I_{2^{n-2}} \otimes R_{2}\right)=I_{2} \otimes$ $\left\{I_{2^{n-1}}, I_{2^{n-2}} \otimes R_{2}\right\}$.

Proof. From equation (6), we can derive

$$
I_{m} \otimes(\mathscr{A} \times \mathscr{C})=\left(I_{m} \otimes \mathscr{A}\right)\left(I_{m} \otimes \mathscr{C}\right) .
$$

We use equation (42) to rewrite $T_{2^{n}}\left(R_{n}\right)$ to

$$
\begin{aligned}
& T_{2^{n}}\left(R_{n}\right)=\left(I_{2^{n-1}} \otimes\left\{I_{2}, R_{n}, I_{2}, R_{n}, \ldots, R_{n}\right\}\right) \\
& \times\left(I_{2^{n-1}} \otimes\left\{I_{2}, I_{2},\left(R_{n}\right)^{2},\left(R_{n}\right)^{2}, \ldots,\left(R_{n}\right)^{2^{n-1}-2}\right\}\right)
\end{aligned}
$$

Using the similar method in Proposition 1, we have

$$
\begin{aligned}
& I_{2^{n-1}} \otimes\left\{I_{2}, I_{2},\left(R_{n}\right)^{2},\left(R_{n}\right)^{2}, \ldots,\left(R_{n}\right)^{2^{n-1}-2}\right\} \\
& =I_{2^{n-2}} \otimes\left\{I_{2^{2}},\left(I_{2} \otimes R_{n-1}\right)^{1}, \ldots,\left(I_{2} \otimes R_{n-1}\right)^{2^{n-2}-1}\right\} \\
& =T_{2^{n-1}}\left(I_{2} \otimes R_{n-1}\right)
\end{aligned}
$$

and

$$
\begin{aligned}
& I_{2^{n-1}} \otimes\left\{I_{2}, R_{n}, I_{2}, R_{n}, \ldots, R_{n}\right\} \\
& =\prod_{j \text { is odd }} C_{n}^{j}\left(R_{n}\right) \\
& =I_{2^{n-2}} \otimes\left(|1\rangle\left\langle 1\left|\otimes R_{n}+\right| 0\right\rangle\langle 0| \otimes I_{2}\right),
\end{aligned}
$$

where $C_{n}^{j}\left(R_{n}\right)$ is shown in equation (25) by replacing the matrix $U$ with $R_{n}$. 


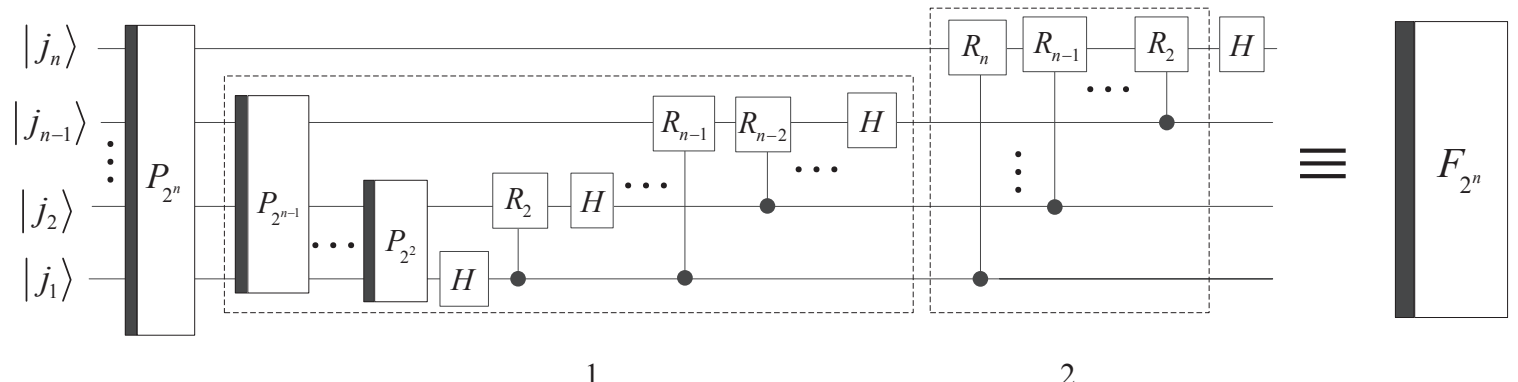

Fig. 7. The first circuit of QFT in (32). The circuits in the dashed box 1 and box 2 implement the QFT $F_{2^{n-1}}$ in (32) and $S_{2^{n}}\left(R_{n}\right)$ in (33), respectively.

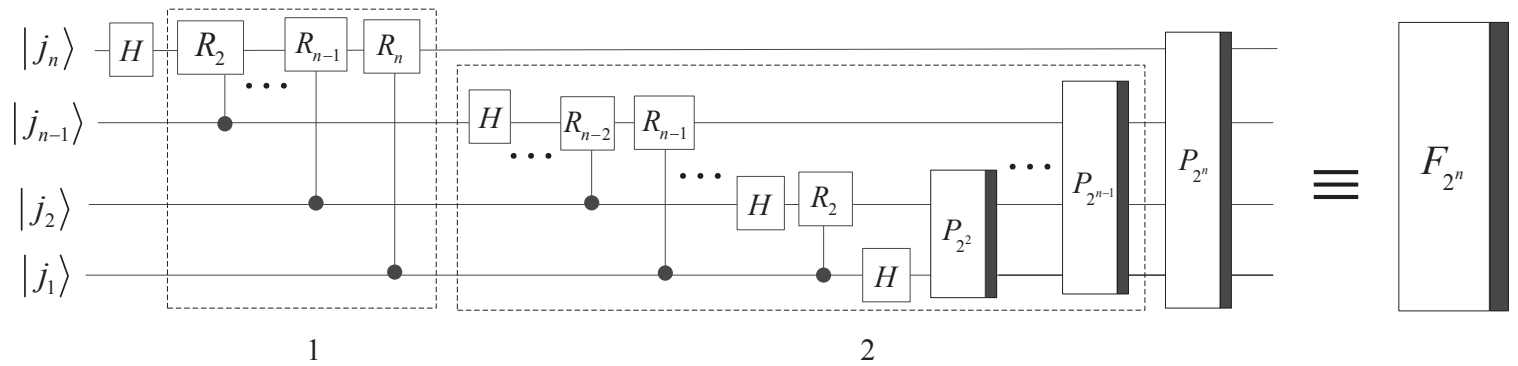

Fig. 8. The second circuit of QFT in (39). The circuits in the dashed box 1 and box 2 implement the QFT $F_{2^{n-1}}$ and $S_{2^{n}}\left(R_{n}\right)$, respectively.

Substituting (44) and (45) into (43), so equation (41) holds. The initial value is given by equation (40)

$$
T_{2^{2}}\left(I_{2^{n-2}} \otimes R_{2}\right)=I_{2} \otimes\left\{I_{2^{n-1}}, I_{2^{n-2}} \otimes R_{2}\right\} .
$$

Thus, the proposition has been proven.

Since QFT is symmetric, we have the following equation by using (3) and (32)

$$
\begin{aligned}
F_{2^{n}}^{3} & =F_{2^{n}}=\left(F_{2^{n}}\right)^{T} \\
& =\left(\left(F_{2^{n-1}}\right) \otimes I_{2}\right) T_{2^{n}}\left(R_{n}\right)\left(I_{2^{n-1}} \otimes H\right) P_{2,2^{n-1}} \\
& =\left(F_{2^{n-1}}^{3} \otimes I_{2}\right) T_{2^{n}}\left(R_{n}\right)\left(I_{2^{n-1}} \otimes H\right) P_{2,2^{n-1}}
\end{aligned}
$$

According to Proposition 2, the third circuit of QFT in (46) with the complexity of $O\left(n^{2}\right)$ is shown in Fig.9.

\section{The fourth circuit of $Q F T$}

Similarly with (46), QFT can be changed to

$$
\begin{aligned}
F_{2^{n}}^{4} & =F_{2^{n}}^{2}=\left(F_{2^{n}}^{2}\right)^{T} \\
& =P_{2^{n-1}, 2}\left(I_{2^{n-1}} \otimes H\right) T_{2^{n}}\left(R_{n}\right)\left(F_{2^{n-1}}^{2} \otimes I_{2}\right) . \\
& =P_{2^{n-1}, 2}\left(I_{2^{n-1}} \otimes H\right) T_{2^{n}}\left(R_{n}\right)\left(F_{2^{n-1}}^{4} \otimes I_{2}\right)
\end{aligned}
$$

By using Proposition 2, the fourth circuit of QFT in (47) with the complexity of $O\left(n^{2}\right)$ is shown in Fig. 10 .

\section{E. The circuits of IQFT}

Comparing (32) with (34), and substituting $R_{n}$ with $\left(R_{n}\right)^{-1}$ in Fig.7, we get the first circuit of IQFT shown in Fig.11.

Similarly, we design the rest three circuits of IQFT by substituting $R_{n}$ with $\left(R_{n}\right)^{-1}$ in Fig.8, Fig.9 and Fig.10. Abbreviation notations of the four circuits of IQFT are shown in Fig.12.

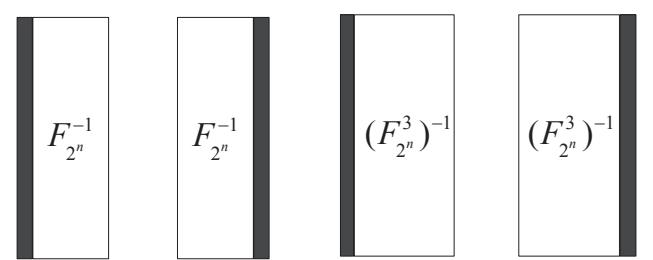

Fig. 12. The abbreviation notations of the circuits of IQFT. From left to right, the abbreviation notations are corresponding to the first, second. third and fourth circuits of IQFT.

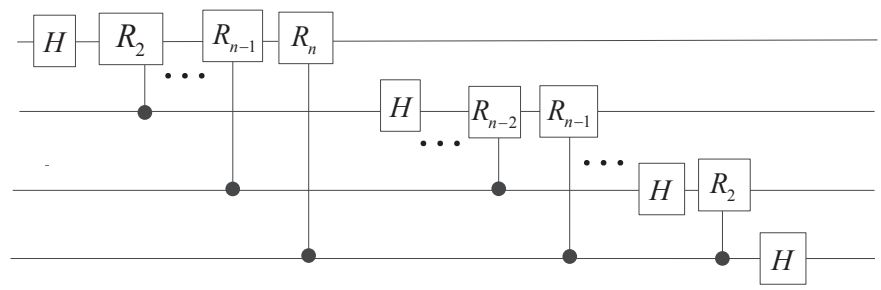

Fig. 13. The circuit of QFT in reference [2].

\section{F. The comparison of circuits of QFT}

In this section, we analyze and compare the circuits in references [2], [26], and [27] with our proposed ones.

Firstly, the circuit in references [2] is shown in Fig.13. We find that it is the second circuit of QFT without the bit reversal, lacking the description of the components in the circuit of QFT, such as box 1 and box2 in Fig.8.

Next, the circuits in references [26] and [27] are shown in Fig.14 and Fig.15, respectively. 


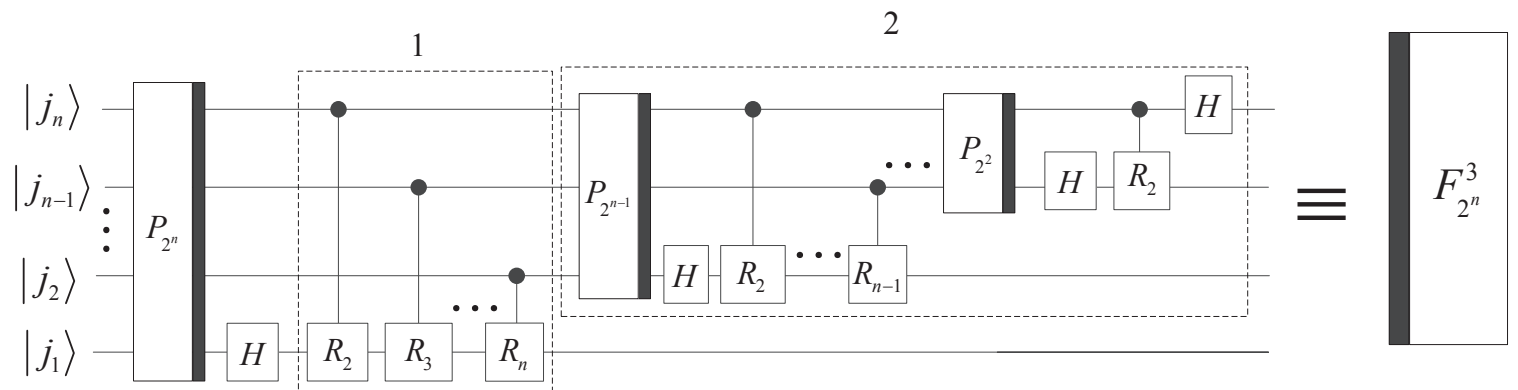

Fig. 9. The third circuit of QFT in (46). The circuits in the dashed box 2 and box 1 implement the QFT $F_{2^{n-1}}$ and $T_{2^{n}}\left(R_{n}\right)$, respectively.

1

2

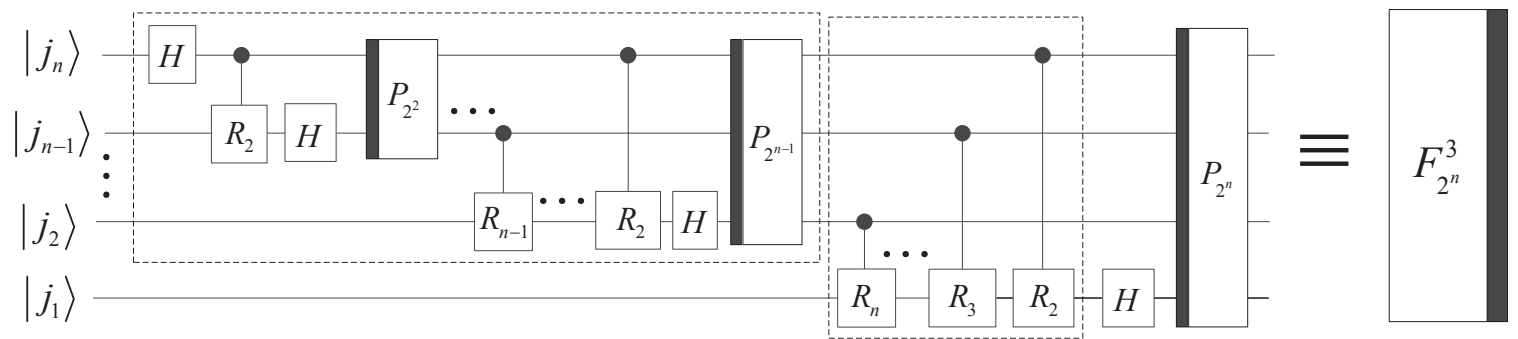

Fig. 10. The fourth circuit of QFT in (47). The circuits in the dashed box 1 and box 2 implement the QFT $F_{2^{n-1}}$ and $T_{2^{n}}\left(R_{n}\right)$, respectively.

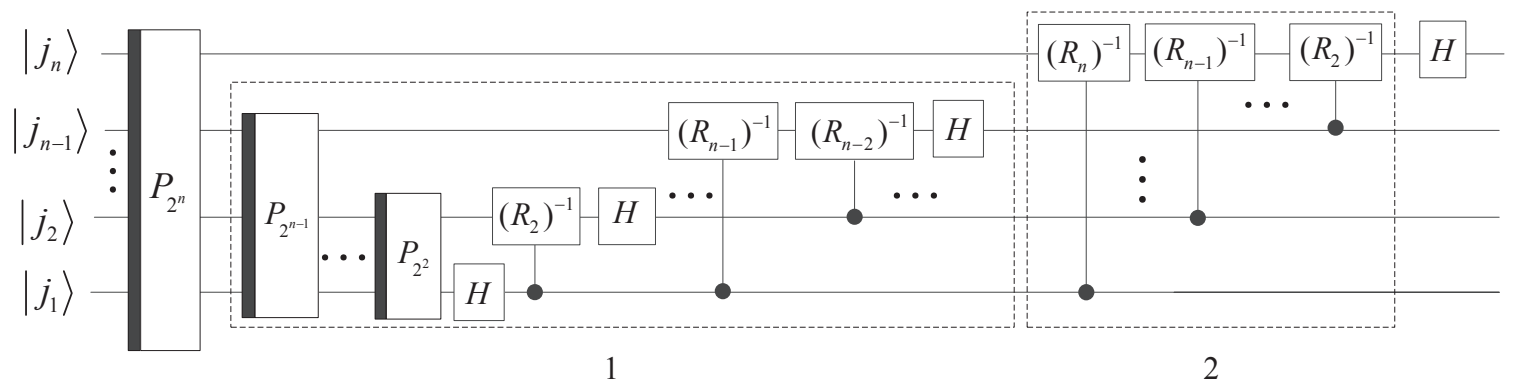

Fig. 11. The first circuit of IQFT. The circuits in the dashed box 1 and box 2 implement the IQFT $\left(F_{2^{n-1}}\right)^{-1}$ and $S_{2^{n}}\left(\left(R_{n}\right)^{-1}\right)$, respectively.

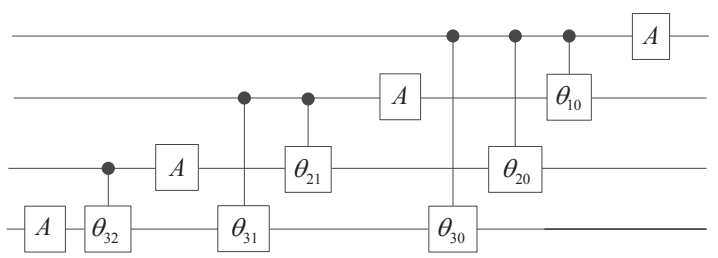

(a)

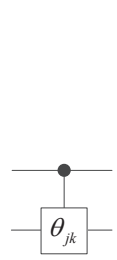

(b)

Fig. 14. The circuit of the QFT in reference [26]. In this figure, $A=H$ and $\theta_{j k}=\frac{\pi}{2^{j-k}}$.

Two gates in (b) of Fig.14 and Fig.15 are both

$$
\left[\begin{array}{cccc}
1 & 0 & 0 & 0 \\
0 & 1 & 0 & 0 \\
0 & 0 & 1 & 0 \\
0 & 0 & 0 & e^{i \theta_{j k}}
\end{array}\right]
$$

and are equal with $C_{1}^{1}\left(R_{j-k+1}\right)$. Therefore, the circuit in (a) of Fig.15 is same with the fourth circuit of QFT without the bit reversal and formula derivation to proves its correctness.

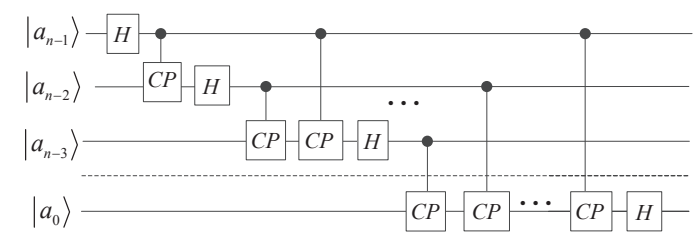

(a)

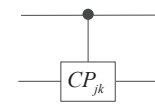

(b)
Fig. 15. The circuit of the QFT in reference [27]. In (b), $C P_{j k}$ symbolizes a controlled phase-shift gate acting on the $j$-th and $k$-th qbits, of which the first is the control and the second the target.

Furthermore, we derive that $C_{1}^{1}\left(R_{j-k+1}\right)=V_{1}^{1}\left(R_{j-k+1}\right)$, thus, the first circuit of the QFT for $n=4$ is redesigned and shown in Fig.16, and the circuit of the dashed box is exactly same with one in Fig.14. Therefore, we can conclude that the circuit of the QFT in the reference [26] is same with the second circuit of the QFT without the bit reversal, lacking formula derivation to proves its correctness.

As mentioned above, we have designed four complete circuits of QFT and IQFT, and described the functions of their 


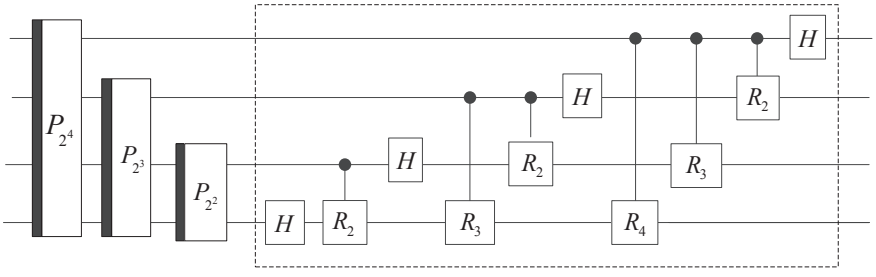

Fig. 16. The first circuit of QFT for $n=4$.

components. Meanwhile, we have proved the correctness of the four complete circuits from their formula derivations.

\section{The 2D AND 3D QFTS BASED ON QVR}

We now design 2D and 3D QFTs, which can be applied on the quantum images and quantum videos, in the section.

\section{A. The 2D QFT based on QVR}

According to MQVR in Section 3, a natural image with size of $2^{n} \times 2^{m}$ can be expressed as an angle matrix

$$
\Lambda_{2^{n}, 2^{m}}=\left[\begin{array}{cccc}
\theta_{0,0} & \theta_{0,1} & \cdots & \theta_{0,2^{m}-1} \\
\theta_{1,0} & \theta_{1,1} & \cdots & \theta_{1,2^{m}-1} \\
\vdots & \vdots & \cdots & \vdots \\
\theta_{2^{n}-1,0} & \theta_{2^{n}-1,1} & \cdots & \theta_{2^{n}-1,2^{m}-1}
\end{array}\right],
$$

where $\theta_{x, y}$ is the color information of the pixel on the coordinate $(x, y)$ and an example is shown in Fig.1. Thus, the 2D DFT on $\Lambda_{2^{n}, 2^{m}}$ is defined as

$$
f t 2\left(\Lambda_{2^{n}, 2^{m}}\right)=F_{2^{n}} \Lambda_{2^{n}, 2^{m}}\left(F_{2^{m}}\right)^{T}=F_{2^{n}} \Lambda_{2^{n}, 2^{m}} F_{2^{m}} .
$$

An image can be stored in the state NASS $\left|\psi_{2}\right\rangle$ in (10) by using a quantum circuit in reference [10]. Suppose that the function $f(\cdot)$ is equivalent to the quantum circuit implementing the storage of the image $\Lambda_{2^{n}, 2^{m}}$, that is,

$$
f\left(\Lambda_{2^{n}, 2^{m}}\right)=\left|\psi_{2}\right\rangle=\left[\begin{array}{c}
B_{1}^{T} \\
\vdots \\
B_{2^{n}}^{T}
\end{array}\right],
$$

where $B_{j}=\left[\begin{array}{llll}\theta_{j, 0} & \theta_{j, 1} & \cdots & \theta_{j, 2^{m}-1}\end{array}\right]$ is the row vector of $\Lambda_{2^{n}, 2^{m}}$ in (48).

Applying the function $f(\cdot)$ on $\Lambda_{2^{n}, 2^{m}} \times F_{2^{m}}$, the result is

$$
f\left(\Lambda_{2^{n}, 2^{m}} F_{2^{m}}\right)=\left[\begin{array}{c}
F_{2^{m}} B_{1}^{T} \\
\vdots \\
F_{2^{m}} B_{2^{n}}^{T}
\end{array}\right] .
$$

From (50) and (51), we have

$$
f\left(\Lambda_{2^{n}, 2^{m}} F_{2^{m}}\right)=\left(I_{2^{n}} \otimes F_{2^{m}}\right) f\left(\Lambda_{2^{n}, 2^{m}}\right)
$$

and

$$
f\left(\Lambda_{2^{n}, 2^{m}}\right)=P_{2^{m}, 2^{n}} f\left(\left(\Lambda_{2^{n}, 2^{m}}\right)^{T}\right) .
$$

Then, we have

$$
\begin{aligned}
& f\left(F_{2^{n}} \Lambda_{2^{n}, 2^{m}}\right) \\
& =P_{2^{m}, 2^{n}} f\left(\left(\Lambda_{2^{n}, 2^{m}}\right)^{T} F_{2^{n}}\right) \\
& =P_{2^{m}, 2^{n}}\left(I_{2^{m}} \otimes F_{2^{n}}\right) P_{2^{n}, 2^{m}} f\left(A_{2^{n}, 2^{m}}\right) \\
& =\left(F_{2^{n}} \otimes I_{2^{m}}\right) f\left(A_{2^{n}, 2^{m}}\right) .
\end{aligned}
$$

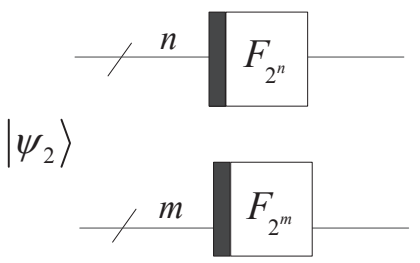

(a) 2D QFT

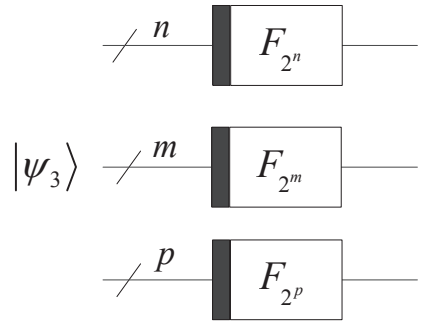

(b) 3D QFT
Fig. 17. The circuits of 2D QFT and 3D QFT.

From equations (49), (52) and (54), the 2D QFT is given by

$$
f t 2\left(\Lambda_{2^{n}, 2^{m}}\right)=f\left(F_{2^{n}} \Lambda_{2^{n}, 2^{m}} F_{2^{m}}\right)=\left(F_{2^{n}} \otimes F_{2^{m}}\right)\left|\psi_{2}\right\rangle
$$

and its quantum circuit is shown in (a) of Fig.17 with the complexity of $O\left(n^{2}+m^{2}\right)$ for a $2^{n} \times 2^{m}$ image.

\section{B. The $3 D Q F T$ based on QVR}

With MQVR in Section 3, a video of $2^{p}$ frames of size $2^{n} \times 2^{m}$ corresponds to the following angle matrix.

$$
A_{2^{n}, 2^{m}, 2^{p}}=\left(\Lambda_{2^{n}, 2^{m}}^{1}, \Lambda_{2^{n}, 2^{m}}^{2}, \cdots, \Lambda_{2^{n}, 2^{m}}^{2^{p}}\right),
$$

where the angle matrix $\Lambda_{2^{n}, 2^{m}}^{k}$ is the $k$-th frame.

We firstly define the following DFTs: $F^{x}(\cdot), F^{y}(\cdot)$ and $F^{t}(\cdot)$ below.

$$
\begin{aligned}
& F^{x}\left(A_{2^{n}, 2^{m}, 2^{p}}\right)=\left(F_{2^{n}} \Lambda_{2^{n}, 2^{m}}^{1}, \cdots, F_{2^{n}} \Lambda_{2^{n}, 2^{m}}^{2^{p}}\right), \\
& F^{y}\left(A_{2^{n}, 2^{m}, 2^{p}}\right)=\left(\Lambda_{2^{n}, 2^{m}}^{1} F_{2^{m}}, \cdots, \Lambda_{2^{n}, 2^{m}}^{2^{p}} F_{2^{m}}\right), \\
& F^{t}\left(A_{2^{n}, 2^{m}, 2^{p}}\right)=\left(C_{2^{n}, 2^{m}}^{1}, C_{2^{n}, 2^{m}}^{2}, \cdots, C_{2^{n}, 2^{m}}^{2^{p}}\right)
\end{aligned}
$$

with the row vectors

$$
\left[\begin{array}{llll}
C_{x, y}^{1} & C_{x, y}^{2} & \cdots & C_{x, y}^{2^{p}}
\end{array}\right]=u_{x, y} \times F_{2^{p}}
$$

and

$$
u_{x, y}=\left[\begin{array}{llll}
\theta_{x, y}^{1} & \theta_{x, y}^{2} & \cdots & \theta_{x, y}^{2^{p}}
\end{array}\right],
$$

where $C_{x, y}^{j}$ and $\theta_{x, y}^{j}$ are the elements of the matrices $C_{2^{n}, 2^{m}}^{j}$ and $\Lambda_{2^{n}, 2^{m}}^{j}$ on the position $(x, y)$, respectively.

Next, the 3D DFT of $A_{2^{n}, 2^{m}, 2^{p}}$ can be defined as

$$
f t 3\left(A_{2^{n}, 2^{m}, 2^{p}}\right)=F^{t}\left(F^{y}\left(F^{x}\left(A_{2^{n}, 2^{m}, 2^{p}}\right)\right)\right) .
$$

Similar to (50), we utilize the equivalent function of the quantum circuit to create the QVP of $A_{2^{n}, 2^{m}, 2^{p}}$, so we have

$$
\begin{aligned}
\left|\psi_{3}\right\rangle= & f\left(A_{2^{n}, 2^{m}, 2^{p}}\right) \\
= & {\left[\begin{array}{llllll}
u_{0,0} & \cdots & u_{0,2^{m}-1} & u_{1,0} & \cdots & u_{1,2^{m}-1} \\
& \cdots & u_{2^{n}-1,0} & \cdots & u_{2^{n}-1,2^{m}-1}
\end{array}\right]^{T}, }
\end{aligned}
$$

where the row vector $u_{x, y}$ is shown in equation (61).

Applying the function $f(\cdot)$ in (63) on $F^{t}\left(A_{2^{n}, 2^{m}, 2^{p}}\right)$, $F^{y}\left(A_{2^{n}, 2^{m}, 2^{p}}\right)$ and $F^{x}\left(A_{2^{n}, 2^{m}, 2^{p}}\right)$ respectively, we have the following three equations:

$$
f\left(F^{t}\left(A_{2^{n}, 2^{m}, 2^{p}}\right)\right)=\left(I_{2^{n+m}} \otimes F_{2^{p}}\right)\left|\psi_{3}\right\rangle,
$$




$$
\begin{aligned}
& f\left(F^{y}\left(A_{2^{n}, 2^{m}, 2^{p}}\right)\right)=\left(I_{2^{n}} \otimes F_{2^{m}} \otimes I_{2^{p}}\right)\left|\psi_{3}\right\rangle, \\
& f\left(F^{x}\left(A_{2^{n}, 2^{m}, 2^{p}}\right)\right)=\left(F_{2^{n}} \otimes I_{2^{m}} \otimes I_{2^{p}}\right)\left|\psi_{3}\right\rangle .
\end{aligned}
$$
QFT

Using equations (62), (64), (65) and (66), we drive the 3D

$$
f\left(f t 3\left(A_{2^{n}, 2^{m}, 2^{p}}\right)\right)=\left(F_{2^{n}} \otimes F_{2^{m}} \otimes F_{2^{p}}\right)\left|\psi_{3}\right\rangle .
$$

That is, the 3D QFT on the videos in equation (56) is $F_{2^{n}} \otimes$ $F_{2^{m}} \otimes F_{2^{p}}$, and its quantum circuit is shown in (b) of Fig.17 with the complexity of $O\left(n^{2}+m^{2}+p^{2}\right)$.

Analyzing the multidimensional (i.e., 2D and 3D) QFT and DFT, we draw the conclusion: the multidimensional QFT can be implemented by parallel unning multiple one-dimension QFTs while the multidimensional DFT executes multiple onedimension DFTs in sequence. It is one of the advantages of the QFT.

\section{Simulation EXPERIMENTS}

In the absence of the quantum computer, experiments on quantum images and videos are simulated in Matlab version 2010a on a classical computer.

Let $A_{g}$ and $A_{c}$ be the $128 \times 128$ and $128 \times 128 \times 3$ matrices of the gray-scale and color images shown in Fig.18 (a), respectively. We can obtain two $128 \times 128$ angle matrices $\Lambda_{g}$ and $\Lambda_{c}$ by using the method in Section 3 , that is,

$$
\Lambda_{g}=\frac{\pi C_{g}}{2 \times 2^{8}-2} A_{g}
$$

and

$$
\Lambda_{c}=\frac{\pi C_{c}}{2 \times 2^{24}-2} A_{t},
$$

where $C_{g}$ and $C_{c}$ are two constants corresponding to the two images, and an element of $128 \times 128$ matrix $A_{t}(x, y)$ can be computed by

$$
\begin{aligned}
A_{t}(x, y)=256 \times 256 \times A_{c}(x, y, 1) & +256 \times A_{c}(x, y, 2)+A_{c}(x, y, 3) .
\end{aligned}
$$

Let $\Lambda=\Lambda_{g}$ or $\Lambda=\Lambda_{c}$, and the NASS state

$$
\left|\psi_{2}\right\rangle=f(\Lambda)
$$

can be regarded as a column vector where the function $f(\cdot)$ is defined in equation (50). Therefore, we have the following equations from (55)

$$
f t 2\left(A_{g}\right)=\frac{2 \times 2^{8}-2}{\pi C_{g}} f^{-1}\left(\left(F_{2^{7}} \otimes F_{2^{7}}\right)\left|\psi_{2}\right\rangle\right)
$$

and

$$
f t 2\left(A_{t}\right)=\frac{2 \times 2^{24}-2}{\pi C_{c}} f^{-1}\left(\left(F_{2^{7}} \otimes F_{2^{7}}\right)\left|\psi_{2}\right\rangle\right),
$$

where $f^{-1}(\cdot)$ is the inverse function of $f(\cdot)$, which converts a column vector into a 2-dimension matrix.

The right parts of equations (72) and (73) are written as the symbols $Q F T 2\left(A_{g}\right)$ and $Q F T 2\left(A_{t}\right)$, respectively. In order to compare our proposed 2D QFT with the 2D inverse DFT (IDFT) $f f t 2(\cdot)$ in Matlab, we introduce the corresponding IDFT

$$
x[k] \rightarrow \frac{1}{2^{n}} \sum_{j=0}^{2^{n}-1} e^{2 \pi i j k / 2^{n}} x[j],
$$

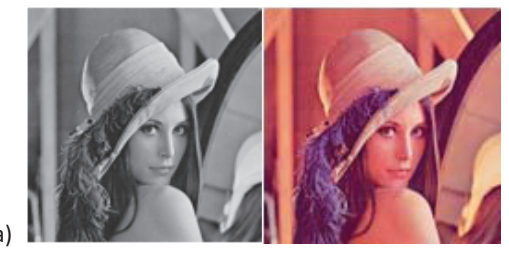

(b)

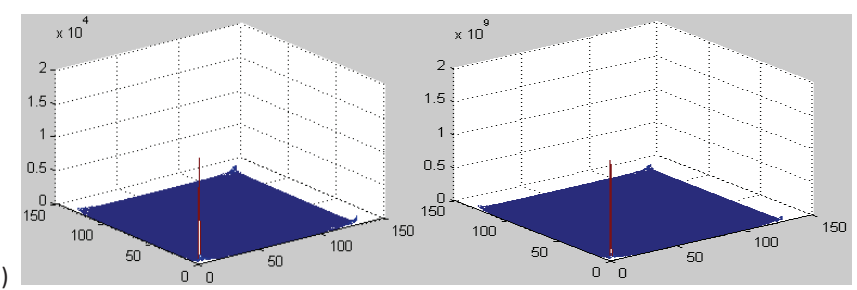

Fig. 18. The 2D QFT on two $128 \times 128$ images. (a) Original images; (b) Amplitude spectrums.

where $x[j]$ is a column vector. Thus, we have

$$
\operatorname{QFT} 2\left(A_{g}\right)=2^{7} \times \text { ifft } 2\left(A_{g}\right)
$$

and

$$
\operatorname{QFT} 2\left(A_{t}\right)=2^{7} \times \text { ifft } 2\left(A_{t}\right) .
$$

Applying the 2D QDF on two images in Fig.18 (a), experimental results are shown in Fig.18 (b) and Table 1. The amplitude spectrums of matrices $Q F T 2\left(A_{g}\right)$ and $Q F T 2\left(A_{t}\right)$ in Fig.18 (b) show that the 2D QDF is useful for quantum images. By analyzing Table I, we conclude that the results are same among ft2, QFT2 and iff2 without consideration of truncation error on machine computing and verify that our proposed 2D QFT holds.

Let $V_{g}$ and $V_{t}$ be the $64 \times 64 \times 4$ matrices of the gray-scale and color videos shown in Fig.19 (a) and Fig.20 (a). Similarly, we obtain the following equations by using equation (67)

$$
f t 3\left(V_{g}\right)=\frac{2 \times 2^{8}-2}{\pi C_{g}} f^{-1}\left(\left(F_{2^{6}} \otimes F_{2^{6}} \otimes F_{2^{2}}\right)\left|\psi_{3}\right\rangle\right)
$$

and

$$
f t 3\left(V_{t}\right)=\frac{2 \times 2^{24}-2}{\pi C_{c}} f^{-1}\left(\left(F_{2^{6}} \otimes F_{2^{6}} \otimes F_{2^{2}}\right)\left|\psi_{3}\right\rangle\right),
$$

where $C_{g}, C_{c}$ are two constants corresponding to the two videos and the right parts of equations (77) and (78) written as $Q F T 3\left(V_{g}\right)$ and $Q F T 3\left(V_{t}\right)$.

Comparing (15) with (74), we have

$$
\operatorname{QFT} 3\left(V_{g}\right)=2^{7} \times \text { ifftn }\left(V_{g}\right)
$$

and

$$
\operatorname{QFT} 3\left(V_{t}\right)=2^{7} \times \text { ifftn }\left(V_{t}\right),
$$

where $\operatorname{fftn}(\cdot)$ is the multidimensional IDFT in Matlab.

Applying the 3D QDF on two videos in Fig.19 (a) and Fig.20 (a), experimental results are shown in Fig.19 (b), Fig.20 (b) and Table 2. The amplitude spectrums of matrices QFT3 $\left(V_{g}\right)$ and $Q F T 3\left(V_{t}\right)$ in Fig.19 (b) and Fig.20 (b) show that the 3D QDF is useful for quantum videos. By analyzing Table II, we conclude that the results are the same among $\mathrm{ft} 3$, QFT3 and iffn without consideration of truncation error on machine computing and verify that our proposed 3D QFT holds. 
TABLE I

COMPARISONS AMONG FT2, QFT2 AND IFF2, WHERE MAVR AND MAVI ARE THE ABBREVIATIONS OF MAX ABSOLUTE VALUE OF THE REAL PART AND THE IMAGINE PART, RESPECTIVELY

(a)
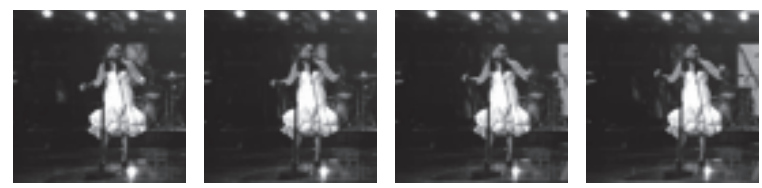

(b)
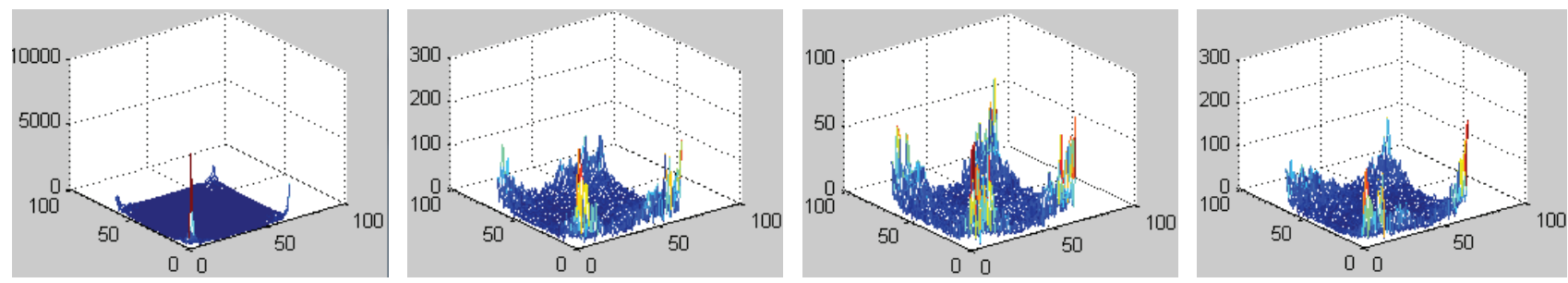

Fig. 19. The 3D QFT on a gray-scale video. (a) The four $64 \times 64$ frames extracted in the video; (b) Amplitude spectrums of the four frames.

(a)

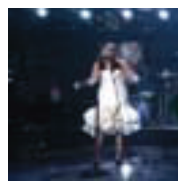

(b)
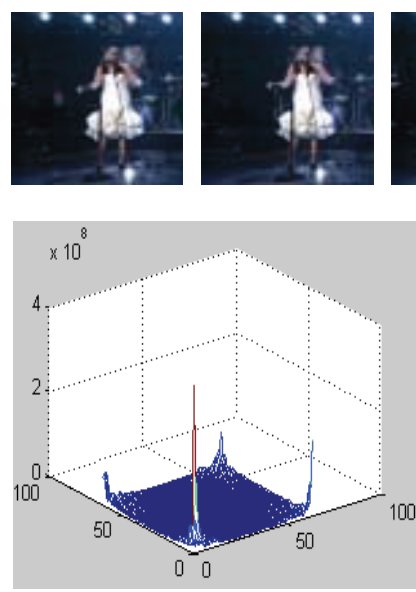
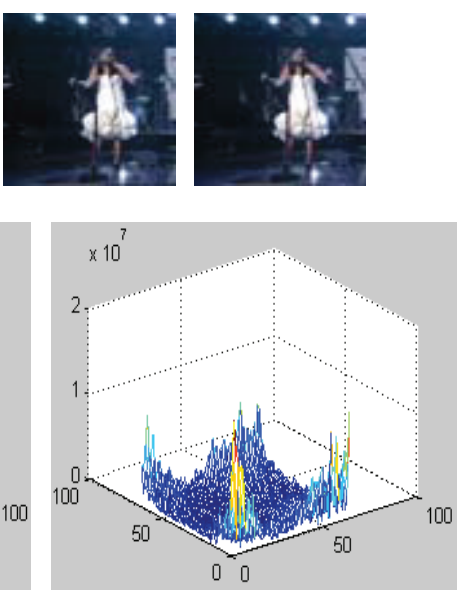
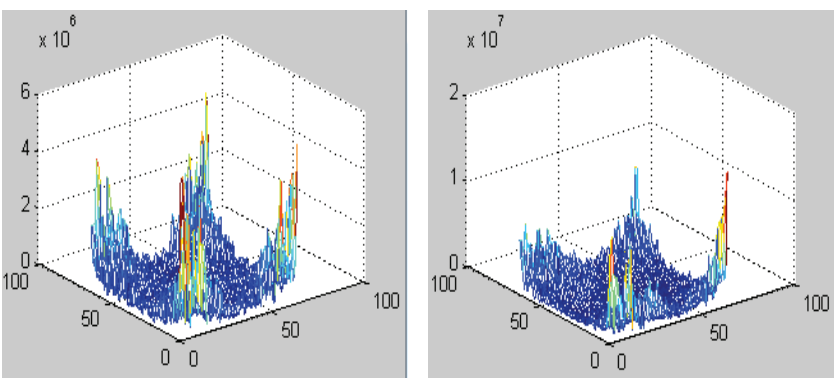

Fig. 20. The 3D QFT on a color video. (a) The four $64 \times 64$ frames; (b) Amplitude spectrums of the four frames.

TABLE II

COMPARISONS AMONG FT3, QFT3 AND IFFN, WHERE MAVR AND MAVI ARE THE SAME BETWEEN TABLE I AND TABLE II

\begin{tabular}{ccccc}
\hline & MAVR of ft3-QFT3 & MAVI of ft3-QFT3 & MAVR of $2^{7} \times$ ifftn-QFT3 & MAVI of $2^{7} \times$ ifftn-QFT3 \\
\hline$V_{g}$ & $7.8217 \times 10^{-11}$ & $3.3197 \times 10^{-11}$ & $7.5488 \times 10^{-11}$ & $3.5016 \times 10^{-11}$ \\
\hline$V_{t}$ & $8.9407 \times 10^{-7}$ & $8.0466 \times 10^{-7}$ & $7.7486 \times 10^{-7}$ & $9.5367 \times 10^{-7}$ \\
\hline
\end{tabular}

\section{CONCLUSION}

In this article, we has described MQVR by extending quantum image representation NASS into QVR, which is the foundation of QVIP. We have also designed four complete circuits of QFT and IQFT on $2^{n}$ elements with complexity $O\left(n^{2}\right)$, and showed the functions of various components of these circuits. Compared with the existing circuits of QFT, our proposed circuits are exactly complete and their correctness have been proved by their formula derivation and simulation experiments. Therefore, our proposed QFTs are the beneficial complement and enhancement of the current circuits. For the first time, we have implemented the 2D and 3D QFTs based on QVR with complexities $\Theta\left(n^{2}+m^{2}\right)$ and $\Theta\left(n^{2}+m^{2}+p^{2}\right)$ for $2^{n} \times 2^{m}$ images and $2^{n} \times 2^{m} \times 2^{p}$ videos, respectively. Furthermore, our proposed 2D and 3D QFTs can be implemented by parallel running multiple one-dimension QFTs while the classical 2D and 3D DFTs execute multiple one-dimension DFTs in sequence. These have proved that our proposed QFTs are efficient. The results of simulation experiments show that our proposed QFTs are valid and useful for QVIP. In summary, 
we have provided a complete framework of QFT and a feasible scheme for QFT to be applied in QVIP.

\section{ACKNOWLEDGMENT}

This work is supported by the National Natural Science Foundation of China under Grant No.61462026 and No.61762012, Project of Science and Technology of Jiangxi province Grant No. 20161BAB202065, the key research project of Guangxi Normal University Grant No.2016ZD008 and an award of China Scholarship Council.

\section{REFERENCES}

[1] J. Stajic, "The future of quantum information processing," Science, vol. 339, pp. 1163-1163, 2013.

[2] M. A. Nielsen, I. L. Chuang, Quantum Computation and Quantum Information, CambridgeCambridge University Press, 2000.

[3] P. W. Shor, "Algorithms for quantum computation: Discrete logarithms and factoring," Foundations of Computer Science, 1994 Proceedings, 35th Annual Symposium on. IEEE, 1994, pp. 124-134.

[4] D. Deutsch, "Quantum theory, the Church-Turing principle and the universal quantum computer," Proceedings of the Royal Society of London A: Mathematical, Physical and Engineering Sciences, 1985, pp. 97-117.

[5] L. Grover, "A fast quantum mechanical algorithm for database search," Proceedings of the 28th Annual ACM Symposium on the Theory of Computing, 1996, pp. 212-219.

[6] G. Beach, C. Lomont, C. Cohen, "Quantum Image Processing (QuIP)," 32nd Applied Imagery Pattern Recognition Workshop, 2003, pp. 39-44.

[7] S. E. Venegas-Andraca, S. Bose, "Quantum computation and image processing: new trends in artificial intelligence," Proceedings of the International Congress on Artificial Intelligence, 2003, pp. 1563-1564.

[8] S. E. Venegas-Andraca, S. Bose, "Storing, processing, and retrieving an image using quantum mechanics," Proceedings of the SPIE Conference on Quantum Information and Computation, 2003, pp. 137-147.

[9] P. Q. Le, F. Dong, K. Hirota, "A flexible representation of quantum images for polynomial preparation, image compression, and processing operations," Quantum Information Processing, vol. 10, pp. 63-84, 2011.

[10] H. S. Li, Q. Zhu, R. G. Zhou, et al., "Multidimensional color image storage, retrieval, and compression based on quantum amplitudes and phases," Information Sciences, vol. 273, pp. 212-232, 2014.

[11] P. Fan, R. G. Zhou, N. Jing, H. S. Li, "Geometric transformations of multidimensional color images based on NASS," Information Sciences, vol. 340, pp. 191-208, 2016.

[12] Y. Zhang, K. Lu, Y. Gao, et al., "NEQR: a novel enhanced quantum representation of digital images," Quantum Information Processing, vol. 12, no. 8, pp. 2283-2860, 2013.

[13] A. Fijany, C. P. Williams, "Quantum wavelet transforms: Fast algorithms and complete circuits," Lect. Notes Comput. Science, vol. 1509, pp. 10-33, 1998.

[14] F. Yan, A. M. Iliyasu, S. E. Venegas-Andraca, et al., "Video encryption and decryption on quantum computers," International Journal of Theoretical Physics, vol. 54, no. 8, pp. 2893-2904, 2015.

[15] F. Yan, A. M. Iliyasu, A. R. Khan, et al., "Measurements-based Moving Target Detection in Quantum Video," International Journal of Theoretical Physics, vol. 55, no. 4, pp. 2162-2173, 2016.

[16] H. S. Li, Q. Zhu, R. G. Zhou, et al., "Multi-dimensional color image storage and retrieval for a normal arbitrary quantum superposition state," Quantum Information Processing, vol. 13, no. 4, pp. 991-1011, 2014.

[17] H. S. Li, Q. Zhu, L. Song, et al., "Image storage, retrieval, compression and segmentation in a quantum system," Quantum Information Processing, vol. 12, no. 6, pp. 2269-2290, 2013.

[18] C. Y. Pang, Z. W. Zhou, G. C. Guo, "Quantum discrete cosine transform for image compression," http://arXiv preprint quant-ph/0601043, 20106.

[19] B. Sun, A. Iliyasu, F. Yan, et al., "An rgb multi-channel representationfor images on quantum computers," Journal of Advanced Computational Intelligence and Intelligent Informatics, vol. 17, no. 3, pp. 404-417, 2013.

[20] S. Yuan, X. Mao, Y. Xue, et al., "SQR: a simple quantum representation of infrared images," Quantum Information Processing, vol. 13, no. 6, pp. 1353-1379, 2014

[21] Y. Zhang, K. Lu, Y. Gao, et al., "A novel quantum representation for log-polar images," Quantum Information Processing, vol. 12, no. 9, pp. 3103-3126, 2013
[22] N. Jiang, W. Wu, L. Wang, et al., "Quantum image pseudocolor coding based on the density-stratified method," Quantum Information Processing, vol. 14, no. 5, pp. 1735-1755, 2015.

[23] J. Sang, S. Wang, Q. Li, "A novel quantum representation of color digital images," Quantum Information Processing, vol. 16, no. 2, pp. 1-14, 2017.

[24] J. Wang, "QRDA: Quantum Representation of Digital Audio," International Journal of Theoretical Physics, vol. 55, no. 3, pp. 1622-1641, 2016.

[25] P. W. Shor, "Polynomial-time algorithms for prime factorization and discrete logarithms on a guantum computer," SIAM Journal on Computing, vol. 26, no. 5, pp. 1484-1509, 1997.

[26] A. Barenco , A. Ekert, K. A. Suominen, et al., "Approximate quantum Fourier transform and decoherence," Physical Review A, vol. 54, no. 1:139, 1996.

[27] I. G. Karafyllidis, "Visualization of the quantum Fourier transform using a quantum computer simulator," Quantum Information Processing, vol. 2, no. 4, pp. 271-288, 2003.

[28] H. F. Wang, A. D. Zhu, S. Zhang, et al., "Simple implementation of discrete quantum Fourier transform via cavity quantum electrodynamics," New Journal of Physics, vol. 13, no. 1:013021, 2013.

[29] J. Heo, M. S. Kang, C. H. Hong, et al., ”Discrete quantum Fourier transform using weak cross-Kerr nonlinearity and displacement operator and photon-number-resolving measurement under the decoherence effect," Quantum Information Processing, vol. 15, no. 12, pp. 4955-4971, 2016.

[30] D. Coppersmith, "An approximate Fourier transform useful in quantum factoring," arXiv preprint quant-ph/0201067, 2002.

[31] R. B. Griffiths, C. S. Niu, "Semiclassical Fourier transform for quantum computation," Physical Review Letters, vol. 76, no. 17:3228, 1996.

[32] J. W. Cooley, J. W. Tukey, "An algorithm for the machine calculation of complex Fourier series," Mathematics of computation, vol. 19, no. 90, pp. 297-301, 1965.

[33] C. S. Park, "2D discrete Fourier transform on sliding windows," IEEE Transactions on Image Processing, vol. 24, no. 3, pp. 901-907, 2015.

[34] X. Y. He, X. Y. Zhou, T. J. Cui, "Fast 3D-ISAR image simulation of targets at arbitrary aspect angles through nonuniform fast Fourier transform (NUFFT)," IEEE Transactions on Antennas and Propagation, vol. 60, no. 5, pp. 2597-2602, 2012.

[35] W. W. Zhang, F. Gao, B. Liu, et al., "A watermark strategy for quantum images based on quantum fourier transform," Quantum Information Processing, vol. 12, no. 2, pp. 793-803, 2013.

[36] Y. G. Yang, X. Jia, P. Xu, et al., "Analysis and improvement of the watermark strategy for quantum images based on quantum Fourier transform," Quantum information processing, vol. 12, no. 8, pp. 27652769, 2013

[37] Y. G. Yang, J. Xia, X. Jia, et al., "Novel image encryption/decryption based on quantum Fourier transform and double phase encoding," Quantum information processing, vol. 12, no. 11, pp. 3477-3493, 2013.

[38] P. Hoyer, "Efficient quantum transforms," arXiv preprint quantph/9702028, 1997.

[39] B. J. Fino, V. R. Algazi, "A unified treatment of discrete fast unitary transforms," SIAM Journal on Computing, vol. 6, no. 4, pp. 700-717, 1977.

[40] G. L.Long, Y. Sun, 'Efficient Scheme for Initializing a quantum register with an arbitrary superposed state," Phys. Rev. A, vol. 4: 014303, 2001.

[41] J. Chiaverini, J. Britton, D. Leibfried, et al., "Implementation of the semiclassical quantum Fourier transform in a scalable system," science, vol. 308, Issue 5724, pp. 997-1000, 2005. 